\begin{tabular}{|l|l|l|l|l|}
\hline Cuadernos I. Geográfica & 25 & pp. 7-24 & Logroño & 1999 \\
\hline
\end{tabular}

\title{
FACTORES EN LA GENERACIÓN DE CRECIDAS EN UNA CUENCA DE CAMPOS ABANDONADOS, PIRINEO CENTRAL ESPAÑOL
}

\author{
J. ARNÁEZZ ${ }^{1}$ \\ C. MARTÍ BONO \\ S. BEGUERÍA ${ }^{2}$ \\ A. LORENTEE ${ }^{2}$ \\ M.P. ERREA ${ }^{2}$ \\ J.M. GARCÍA RUIZ ${ }^{2}$
}

\begin{abstract}
RESUMEN. Se ban estudiado los factores que explican la variabilidad temporal de los picos de crecida en una cuenca experimental del Pirineo Central. Esta cuenca (Arnás, Valle de Borau) fue completamente cultivada basta hace unos 50 años y se caracteriza por una respuesta bidrológica casi inmediata frente a cualquier intensidad y volumen de precipitación. Se ha comprobado que la cantidad total de precipitación durante cada evento apenas explica la intensidad de los picos de crecida, que muestran una variabilidad muy alta. Las relaciones entre precipitación y caudal mejoran sensiblemente si se separan las crecidas de estación fria y de estación cálida. La lluvia registrada durante 3 y 7 dias antes de cada crecida tiene un papel relativamente importante en invierno. Durante el verano $y$ principio de otoño la intensidad de los picos de crecida no se relaciona con ninguna variable pluviométrica, debido a la fuerte evapotranspiración y a la separación existente entre eventos lluviosos.
\end{abstract}

ABSTRACT. Factors explaining the temporal variability of peak flows in an experimental catchment of the Central Pyrenees have

1. Area de Geografía, Dpto. de Ciencias Humanas y Sociales, Universidad de La Rioja, 26004-Logroño.

2. Instituto Pirenaico de Ecología, CSIC, Campus de Aula Dei, Apartado 202, 50080Zaragoza. 
J. ARNÁEZ - C. MARTÍ BONO - S. BEGUERÍA - A. LORENTE - M.P. ERREA - J.M. GARCÍA RUIZ

been studied. This catchment (Arnás, Borau Valley) was cultivated until 50 years ago and is now abandoned; it is characterized by a quick bydrological response against any intensity and volume of precipitation. The total volume of precipitation during each rainfall event bardly explains the intensity of peak flows, which show a bigh variability. Relationships between precipitation and discharge very much improve if cold and bot season floods are considered separately. Rainfall recorded during 3 and 7 days before each flood bas a relatively important role in winter. During summer and the beginning of autumn the intensity of peak flows are not related to any pluviometric variable, due to evapotranspiration and the separation between rainstorm events.

Palabras clave: Crecidas, Picos de crecida, Relaciones precipitación-caudal, Intensidad de precipitación, Cuenca experimental, Campos abandonados, Pirineo Central español.

Keywords: Floods, Peak-flows, Relationships precipitation-discharge, Intensity of precipitation, Experimental catchment, Abandoned fields, Central Spanish Pyrenees.

\section{Introducción}

Las respuestas del caudal frente a las precipitaciones varían mucho de unas cuencas a otras en función no sólo de las características de los eventos lluviosos, sino también debido a la densidad de la cubierta vegetal, la profundidad y permeabilidad de los suelos, el tipo de sustrato rocoso y los rasgos de la topografía, especialmente la pendiente (ver, por ej., Dunne y Leopold, 1978). La variabilidad de la respuesta hidrológica es también muy grande dentro de una misma cuenca a lo largo del tiempo, de manera que para una misma precipitación el pico de crecida y la forma del hidrograma pueden ser muy diferentes en distintas épocas del año. Este fenómeno explica la dificultad de la modelización hidrológica, al no existir una relación lineal entre precipitación y caudal. Por otra parte, es bien conocido el hecho de que la intensidad puede variar mucho a lo largo de un evento lluvioso, lo que tiene grandes repercusiones hidrológicas en el caso de las cuencas pequeñas (inferiores a $10 \mathrm{Km}^{2}$ ), mientras que en las mayores esas fluctuaciones de intensidad pasan prácticamente desapercibidas.

En los últimos años el equipamiento de cuencas experimentales en España ha permitido el estudio detallado de procesos hidrológicos y geomorfológicos, así como: sus relaciones con diferentes factores (intensidad de las precipitaciones, humedad del suelo, procesos de erosión en laderas, entre otros) (García Ruiz y Gallart, 1997). Buena parte de tales estudios se han centrado en el análisis de la 
producción y transporte de sedimentos (por ej., Martínez Castroviejo et al., 1991; Batalla et al., 1995; González et al., 1997; Llorens et al., 1997; White et al., 1997; Regüés et al., 1998; Arnáez et al., 1998). Otros trabajos, los menos, se han enfocado hacia problemas más puramente hidrológicos (por ej., Camarasa, 1990; Llorens, 1993; Gallart et al., 1994; Gallart y Llorens, 1996; Gallart et al., 1998; Ceballos y Schnabel, 1998), especialmente en las relaciones entre eventos pluviométricos y respuesta del caudal.

El objetivo de este trabajo es identificar y jerarquizar los factores que explican la diferente respuesta de las avenidas en una cuenca intensamente perturbada por actividades humanas y que actualmente se encuentra en fase de recolonización vegetal. Se ha podido comprobar que la cuenca experimental de Arnás (valle de Borau, Pirineo Central) responde de manera casi inmediata a cualquier registro pluviométrico, facilitando así el estudio de las relaciones entre precipitación y picos de crecida. Estudios precedentes realizados en esta misma cuenca han puesto de manifiesto que tiene una respuesta rápida frente a las precipitaciones y que la recolonización vegetal tras el abandono agrícola tiende a limitar las fuentes de sedimento a las proximidades de los cauces (Arnáez et al., 1998; Lorente et al., en prensa). En 1997 las avenidas representaron el $23 \%$ de la salida total de agua de la cuenca, el $17 \%$ de los solutos, el $81 \%$ del sedimentos en suspensión y el $100 \%$ de la carga de fondo (Arnáez et al., 1998).

\section{El área de estudio}

La cuenca de Arnás se localiza en la cabecera del valle de Borau, dentro del alto valle del río Aragón, en el Pirineo Central (Fig.1). Ocupa una superficie de 284 ha. Su altitud máxima se alcanza a $1330 \mathrm{~m}$, y la inferior a $900 \mathrm{~m}$. Toda la cuenca se instala en el sector del flysch eoceno, con delgados estratos alternantes de areniscas y margas buzando hacia el norte.

El barranco de Arnás circula de manera subsecuente de oeste a este dando lugar a un fuerte contraste entre la ladera solana, correspondiente, a grandes rasgos, a un abrupto frente de cuesta, y la ladera umbría, de pendiente más suave, coincidiendo con un prolongado reverso de cuesta.

La precipitación anual es de alrededor de $900 \mathrm{~mm}$ en la parte baja de la cuenca y, de acuerdo con las regresiones establecidas en otros trabajos (García Ruiz et al., 1985), debe estar próxima a $1300 \mathrm{~mm}$ en las divisorias más elevadas. El periodo más lluvioso ocurre entre noviembre y mayo. La temperatura media anual es de $9-10^{\circ} \mathrm{C}$.

En la ladera umbría predomina un suelo de la clase kastanozem háplico, profundo y bien estructurado, con elevado contenido de materia orgánica y buena permeabilidad. La ladera solana está ocupada por un suelo del tipo regosol calcáreo, muy delgado, intensamente erosionado en las convexidades y con drena- 

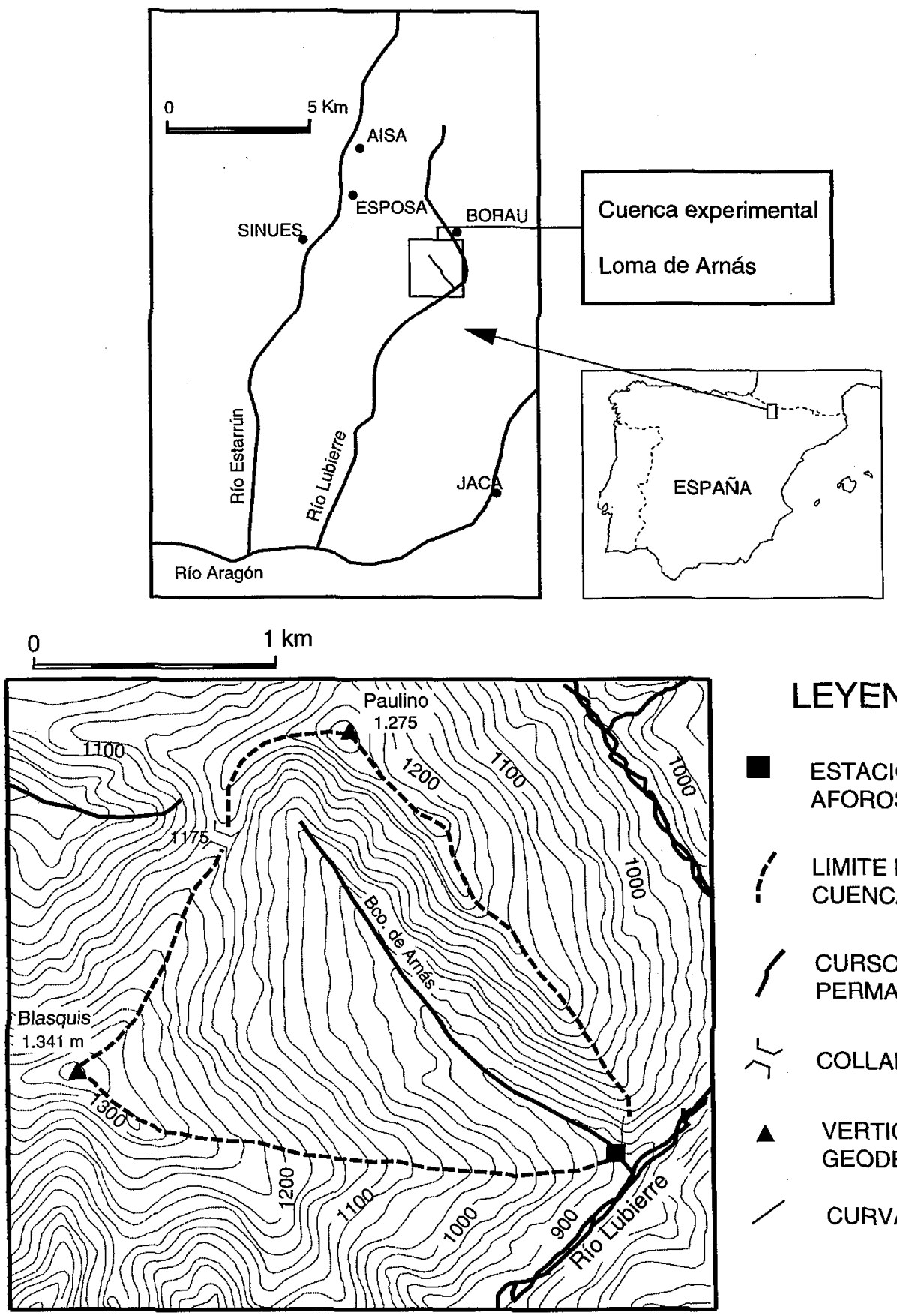

\section{LEYENDA}

- ESTACION DE AFOROS LIMITE DE LA
CUENCA

CURSO DE AGUA PERMANENTE

$\hat{\sim}$ COLLADO

A VERTICE GEODESICO

CURVA DE NIVEL

Fig. 1. Área de estudio (Study area).

je moderado. Las medidas de infiltración oscilan entre 0.25 y $0.75 \mathrm{~mm} \mathrm{~min}^{-1}$, registrándose los valores más bajos en la ladera solana (García Ruiz et al., 1999).

En la actualidad la mayor parte de la cuenca se halla cubierta por matorrales de Genista scorpius y Buxus sempervirens, acompañados de Juniperus communis 
y Rosa gr. canina. Los sectores más elevados de la ladera umbría están colonizados por ejemplares dispersos de Pinus sylvestris y Quercus faginea. Hasta 1950 toda la cuenca estuvo cultivada (principalmente con cereales), incluso en laderas muy pendientes y de formas convexas de la vertiente solana.

\section{Equipamiento y métodos}

A la salida de la cuenca de Arnás se ha construido un flume y se ha instalado el siguiente equipamiento:

- Sensor de ultrasonidos para la medición continua de la altura del agua (Lundhal DCU-7110), controlada anteriormente mediante un sensor de presión.

- Turbidímetro (LYX 800 PT1) para la medición continua de la turbidez del agua, posibilitando así el cálculo de la concentración de sedimento en suspensión.

- Conductivímetro para la medición continua de la conductividad eléctrica del agua.

- Estación meteorológica equipada con los siguientes sensores: termómetro, higrómetro, anemómetro, piranómetro y pluviómetro.

Todos los instrumentos están conectados a data loggers, que toman la información cada 5 segundos, aunque sólo almacenan el promedio de 5 minutos. Los datos son descargados periódicamente mediante un ordenador portátil.

El funcionamiento de los aparatos ha sido muy irregular. Durante 1996 y 1997 la información sobre precipitación, caudal y transporte de sedimento puede considerarse de buena calidad, a pesar de varios periodos cortos con datos insuficientes. El año 1998 cuenta con un registro incompleto por problemas en los datos de caudal (fallos en el sensor de presión hasta su sustitución por un sensor de ultrasonidos). Desde el inicio de 1999 la cuenca aporta información continua y de excelente calidad, excepto la proporcionada por el conductivímetro.

Con los datos disponibles de 1996 y 1997 se han identificado 39 picos de crecida. Para ello se ha considerado como crecida todo incremento de caudal que supera al menos en tres veces el caudal previo a la tormenta. Para cada crecida se han calculado los siguientes parámetros:

- Precipitación total $(\mathrm{mm})$

- Intensidad máxima de precipitación en 30 y en 60 minutos (mm).

- Pico de caudal $\left(1 \mathrm{~s}^{-1}\right)$.

- Pico de escorrentía directa (pico de caudal, deducido el flujo de base, en $1 \mathrm{~s}^{-1}$ ).

- Caudal medio durante la crecida $\left(1 \mathrm{~s}^{-1}\right)$.

- Tiempo de respuesta (time lag), es decir la diferencia en minutos entre el inicio de la precipitación y el pico de crecida.

- Volumen total de agua $\left(\mathrm{m}^{3}\right)$.

- Coeficiente de escorrentía (\%). 
- Precipitación registrada durante 3,7,15 y 21 días antes de cada crecida.

Con esta información se ha aplicado un Análisis de Varianza (Anova) para definir los rasgos más destacados de las crecidas y se han elaborado matrices de correlación, utilizando posteriormente los residuales de algunas de las regresiones como variables dependientes. Una regresión múltiple paso a paso ha permitido identificar los factores más directamente implicados en la mayor o menor intensidad de las crecidas.

\section{Resultados}

La Tabla 1 incluye información sobre la precipitación total ( $\mathrm{mm})$, el pico de crecida y la intensidad de la precipitación en 30 minutos para cada una de las 39 crecidas identificadas durante el periodo de estudio. El pico más elevado se registró el 21 de enero de 1996, con $18831 \mathrm{~s}^{-1}$, correspondiente a una precipitación de $61.2 \mathrm{~mm}$.

Tabla 1. Características generales de las crecidas en la cuenca de Arnás

\begin{tabular}{|rcccc|}
\hline Fecha & $\begin{array}{c}\text { Precipitación } \\
\text { Total } \\
(\mathrm{mm})\end{array}$ & $\begin{array}{c}\text { Caudal } \\
\text { máximo } \\
(1 / \mathrm{s})\end{array}$ & $\begin{array}{c}\text { Precipitación } \\
\text { máxima en } \\
30 \text { minutos }\end{array}$ \\
\hline 1 & $1 / 01 / 96$ & 8,0 & 101,20 & 1,6 \\
2 & $5 / 1 / 96 \mathrm{a}$ & 9,0 & 119,41 & 3,0 \\
3 & $5 / 1 / 96 \mathrm{~b}$ & 14,6 & 366,55 & 3,4 \\
4 & $6 / 01 / 96$ & 6,2 & 164,37 & 2,2 \\
5 & $7 / 01 / 96$ & 5,2 & 118,12 & 0,8 \\
6 & $9 / 01 / 96$ & 36,2 & 905,41 & 2,8 \\
7 & $21 / 01 / 96$ & 61,2 & 1883,79 & 8,6 \\
8 & $1 / 04 / 96$ & 14,2 & 198,37 & 3,2 \\
9 & $16 / 04 / 96$ & 18,0 & 113,66 & 4,4 \\
10 & $6 / 05 / 96$ & 36,0 & 207,66 & 3,0 \\
11 & $7 / 05 / 96$ & 5,6 & 120,03 & 2,2 \\
12 & $9 / 05 / 96$ & 9,8 & 92,81 & 8,0 \\
13 & $10 / 05 / 96$ & 11,2 & 193,81 & 1,6 \\
14 & $18 / 5 / 96 \mathrm{a}$ & 9,2 & 96,44 & 5,6 \\
15 & $18 / 5 / 96 \mathrm{~b}$ & 7,2 & 162,97 & 3,2 \\
16 & $19 / 05 / 96$ & 9,6 & 176,03 & 3,2 \\
17 & $7 / 08 / 96$ & 29,2 & 191,75 & 9,2 \\
18 & $20 / 01 / 97$ & 4,6 & 439,78 & 1,0 \\
19 & $22 / 01 / 97$ & 8,0 & 82,63 & 1,8 \\
\hline
\end{tabular}


FACTORES EN LA GENERACIÓN DE CRECIDAS EN UNA CUENCA DE CAMPOS ABANDONADOS

\begin{tabular}{|ccccc|}
\hline Fecha & $\begin{array}{c}\text { Precipitación } \\
\text { Total } \\
(\mathrm{mm})\end{array}$ & $\begin{array}{c}\text { Caudal } \\
\text { máximo } \\
(1 / \mathrm{s})\end{array}$ & $\begin{array}{c}\text { Precipitación } \\
\text { máxima en } \\
30 \text { minutos }\end{array}$ \\
\hline 20 & $14 / 02 / 97$ & 27,6 & 122,00 & 3,6 \\
21 & $16 / 07 / 97$ & 35,0 & 1716,24 & 9,4 \\
22 & $22 / 07 / 97$ & 7,4 & 116,18 & 1,8 \\
23 & $23 / 07 / 97$ & 20,8 & 767,19 & 20,6 \\
24 & $28 / 07 / 97$ & 11,0 & 139,90 & 8,8 \\
25 & $4 / 08 / 97$ & 27,6 & 81,54 & 17,8 \\
26 & $10 / 08 / 97$ & 16,6 & 89,27 & 3,6 \\
27 & $1 / 09 / 97$ & 25,4 & 142,00 & 15,6 \\
28 & $6 / 11 / 97$ & 9,4 & 167,21 & 1,6 \\
29 & $7 / 11 / 97$ & 17,0 & 167,21 & 1,6 \\
30 & $8 / 11 / 97$ & 25,2 & 678,56 & 3,4 \\
31 & $10 / 11 / 97$ & 36,4 & 800,07 & 3,0 \\
32 & $18 / 11 / 97$ & 14,0 & 106,76 & 1,8 \\
33 & $29 / 11 / 97$ & 21,8 & 161,94 & 2,2 \\
34 & $30 / 11 / 97$ & 3,4 & 188,56 & 2,4 \\
35 & $2 / 12 / 97$ & 11,4 & 372,89 & 3,6 \\
36 & $16 / 12 / 97$ & 56,2 & 697,81 & 4,2 \\
37 & $17 / 12 / 97$ & 28,9 & 1864,99 & 2,6 \\
38 & $18 / 12 / 97$ & 37,0 & 688,50 & 8,6 \\
39 & $31 / 12 / 97$ & 19,0 & 287,12 & 1,2 \\
\hline & & & &
\end{tabular}

La Fig. 2 muestra dos ejemplos de crecidas en la cuenca de Arnás, coincidiendo con dos eventos pluviométricos de diferentes características. En el primero de ellos (2.12.97) la lluvia responde a un modelo muy simple de intensidad moderada, con incremento hacia el final. En consecuencia, el hidrograma de crecida muestra también una forma muy sencilla, con fase ascendente relativamente brusca y fase descendente más suave. En el segundo (17.12.1997) la precipitación tuvo una intensidad muy superior durante dos breves periodos de 5 minutos. El hidrograma refleja que el caudal respondió a ambos impulsos, dando lugar a dos picos de crecida, con ascensos muy bruscos.

Debido a que en el área mediterránea la precipitación muestra una fuerte estacionalidad (con intensidades muy diferentes), se ha realizado un análisis de varianza para las crecidas de estación cálida (mayo-octubre) y estación fría (noviembre-abril). Los resultados se incluyen en la Tabla 2, en la que sólo 4 variables muestran diferencias significativas.

Durante 1996 y 1997 la intensidad de la lluvia en 30 y 60 minutos fue mayor en estación cálida, debido a las tormentas de verano, especialmente activas en 
J. ARNÁEZ - C. MARTÍ BONO - S. BEGUERÍA - A. LORENTE - M.P. ERREA - J.M. GARCÍA RUIZ

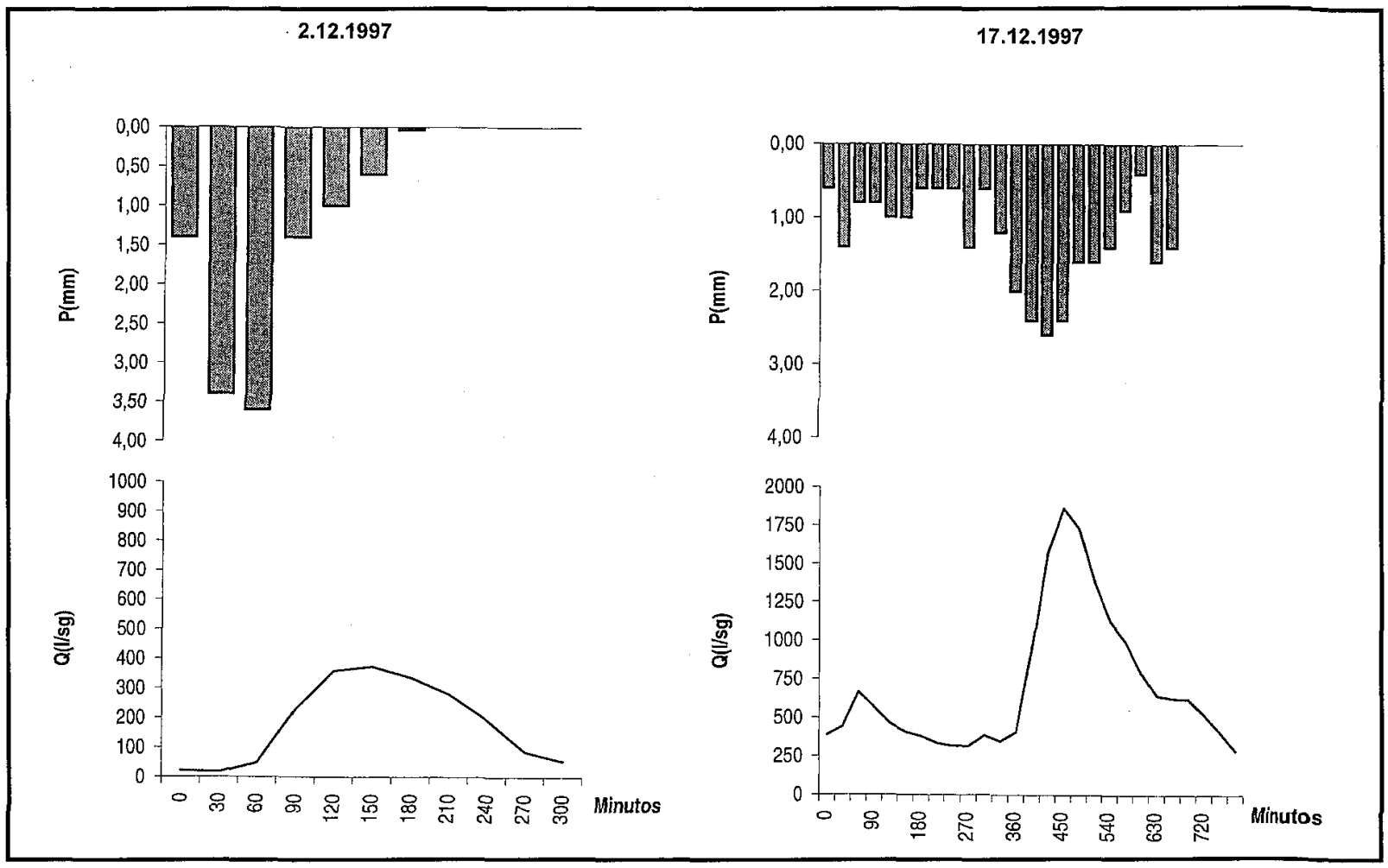

Fig. 2. Ejemplos de crecidas en la cuenca de Arnás (Examples of floods in the Arnás catchment).

junio, julio y agosto de 1996. El tiempo de respuesta del caudal frente a la precipitación es superior en estación fría que en la cálida, sin duda en razón de la diferente intensidad de las tormentas. Por otro lado, el coeficiente de escorrentía es claramente superior en estación fría debido a la menor evapotranspiración y al estado de humedad del suelo. De hecho, los estudios realizados sobre la evolución de la humedad del suelo (Fig. 3) en la cuenca de Arnás confirman que los valores más elevados se concentran durante la estación fría y principios de la cálida, mientras que al final de esta última se registran las cifras más bajas (García Ruiz et al., 1999). Finalmente, conviene señalar que otras dos variables (caudal medio durante la crecida y volumen total de agua) muestran diferencias significativas entre estación fría y estación cálida a nivel de significación del $90 \%$. En ambos casos son mucho mayores durante la estación fría, con lluvias más prolongadas y voluminosas.

La correlación entre precipitación y pico de crecida es baja $\left(\mathrm{r}^{2}=0.33 ; \mathrm{p}=<0.001\right)$ (Fig.4). Este resultado tan pobre significa que, aunque la precipitación total de un evento lluvioso es un factor importante para explicar los picos de crecida, la intensidad de la respuesta hidrológica está también controlada por otros factores. De hecho, pueden registrarse picos de caudal de variado nivel casi con independencia del volumen total de precipitación. Ninguna de las restantes variables 
Tabla 2. Análisis de varianza de las crecidas del barranco de Arnás en 1996 y 97

\begin{tabular}{|c|c|c|c|c|}
\hline Variable & Estación fría & Estación cálida & F-test & Nivel signif. \\
\hline V1 & 20.5 & 17.4 & 0.5 & 0.496 \\
\hline V2 & 3.1 & 7.6 & 11.4 & 0.001 \\
\hline V3 & 4.5 & 9.0 & 8.8 & 0.005 \\
\hline V4 & 24.9 & 13.1 & 2.8 & 0.101 \\
\hline V5 & 40.6 & 33.9 & 2.0 & 0.171 \\
\hline V6 & 66.0 & 60.9 & 0.1 & 0.729 \\
\hline V7 & 90.9 & 75.6 & 1.1 & 0.309 \\
\hline V8 & 187.0 & 101.4 & 4.0 & 0.052 \\
\hline V9 & 450.0 & 505.1 & 1.1 & 0.304 \\
\hline V10 & 408.1 & 239.2 & 1.2 & 0.286 \\
\hline V11 & 11278.6 & 5778.8 & 2.9 & 0.098 \\
\hline V12 & 222.5 & 131.0 & 4.3 & 0.045 \\
\hline V13 & 68.3 & 44.0 & 8.8 & 0.005 \\
\hline
\end{tabular}

Total de crecidas: 39

Crecidas en estación fría: 24

Crecidas en estación cálida: 15

V1: Precipitación total (mm)

V2: Precipitación máxima en 30 minutos

V3: Precipitación máxima en 60 minutos

V4: Precipitación 3 días antes de la crecida $(\mathrm{mm})$

V5: Precipitación 7 días antes de la crecida $(\mathrm{mm})$

V6: Precipitación 15 días antes de la crecida (mm)

V7: Precipitación 21 días antes de la crecida $(\mathrm{mm})$

V8: Caudal medio $(1 / \mathrm{sg})$

V9: Pico de caudal $(1 / \mathrm{sg})$

V10: Pico de escorrentía directa $(1 / \mathrm{sg})$

V11: Volumen total de agua (m3)

V12: Tiempo de respuesta del caudal (minutos)

V13: Coeficiente de escorrentía (\%)

incluidas en la tabla 3 presenta significación estadística, lo que quiere decir que, de forma individual, ni la intensidad de la lluvia ni la lluvia que precede a cada crecida contribuyen a explicar el pico de caudal. 
J. ARNÁEZ - C. MARTÍ BONO - S. BEGUERÍA - A. LORENTE - M.P. ERREA - J.M. GARCÍA RUIZ

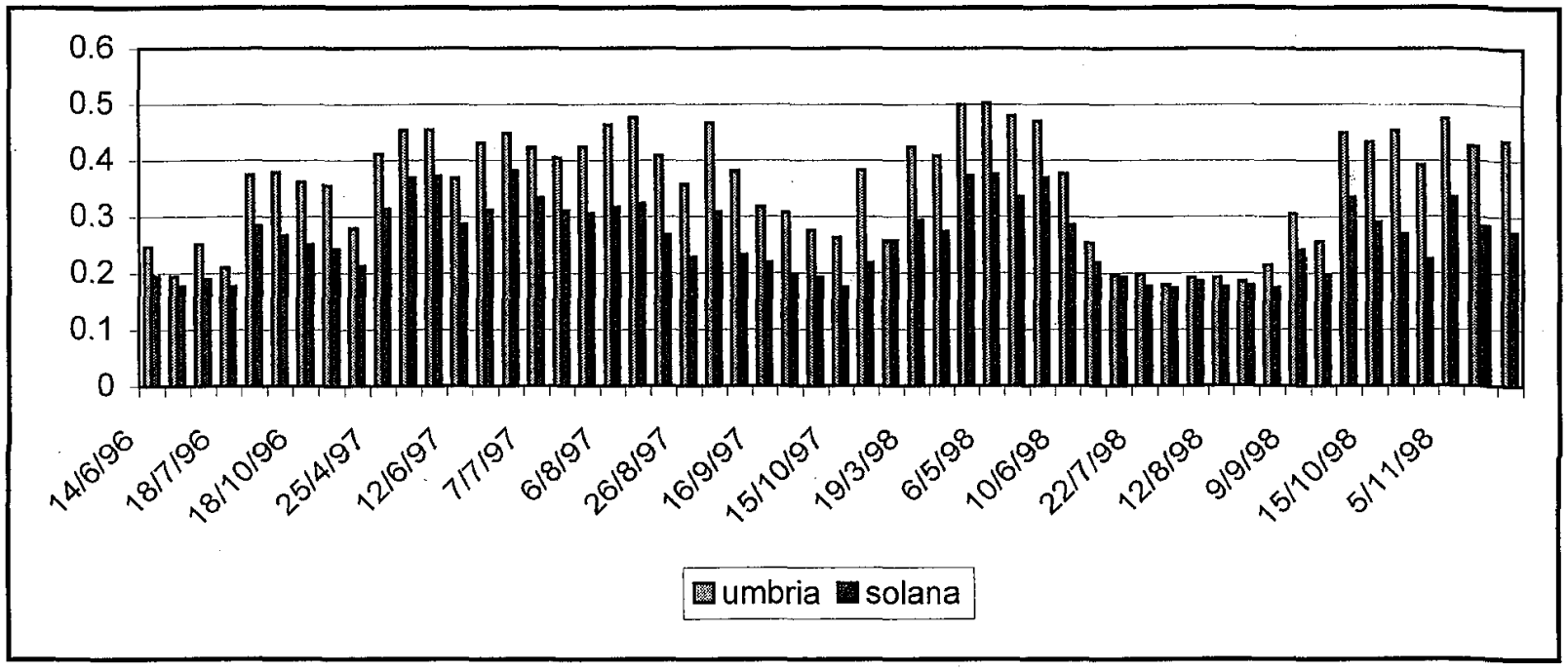

Fig. 3. Evolución de la bumedad del suelo en la cuenca de Arnás en 1996 y 1997, distinguiendo entre la ladera solana y la umbria. Los datos corresponden a la media de 25 puntos de muestro (Evolution of soil humidity in the Arnás catchment during 1996 and 1997. The resulting values correspond to the average of 25 sampling points).

Tabla 3. Coeficientes de determinación y niveles de significación entre el pico de caudal y distintas variables pluviométricas

Variable dependiente: Pico de caudal (1/sg)

\begin{tabular}{|c|c|c|c|c|c|c|}
\hline & \multicolumn{2}{|c|}{ Todos los eventos } & \multicolumn{2}{c|}{ Estación fría } & \multicolumn{2}{c|}{ Estación cálida } \\
\hline & $\mathrm{r}^{2}$ & $\mathrm{p}$ & $\mathrm{r}^{2}$ & $\mathrm{p}$ & $\mathrm{r}^{2}$ & $\mathrm{p}$ \\
\hline V1 & 0.33 & $<0.001$ & 0.41 & $<0.001$ & 0.20 & 0.092 \\
\hline V2 & 0.04 & 0.234 & 0.23 & 0.019 & 0.07 & 0.328 \\
\hline V3 & 0.07 & 0.115 & 0.33 & 0.003 & 0.05 & 0.408 \\
\hline V4 & 0.10 & 0.078 & 0.23 & 0.032 & 0.02 & 0.691 \\
\hline V5 & 0.12 & 0.065 & 0.30 & 0.022 & 0.01 & 0.703 \\
\hline V6 & 0.03 & 0.417 & 0.13 & 0.186 & 0.01 & 0.724 \\
\hline V7 & 0.04 & 0.316 & 0.15 & 0.158 & 0.15 & 0.210 \\
\hline
\end{tabular}

Todas las variables han sido reconvertidas a sus correspondientes valores logarítmicos.

V1: Precipitación total $(\mathrm{mm})$

V2: Precipitación máxima en 30 minutos

V3: Precipitación máxima en 60 minutos

V4: Precipitación 3 días antes de la crecida (mm) 
V5: Precipitación 7 días antes de la crecida $(\mathrm{mm})$ V6: Precipitación 15 días antes de la crecida $(\mathrm{mm})$ V7: Precipitación 21 días antes de la crecida (mm)

La relación mejora sensiblemente -aunque de forma insuficiente- si se consideran por separado las crecidas de estación fría y de estación cálida. En el primer caso la precipitación causante de cada crecida produce un coeficiente de determinación $\left(\mathrm{r}^{2}\right)$ de $0.41(\mathrm{p}=<0.001)$, y además la intensidad de la precipitación (en 30 y 60 minutos) y la lluvia caída 3 y 7 días antes de la crecida proporcionan valores de regresión significativos. En el caso de las crecidas de estación cálida, los resultados son muy frustrantes, con un $\mathrm{r}^{2}$ de sólo $0.20(\mathrm{p}=0.092)$ para la precipitación total y aún menores para el resto de las variables.

Conviene destacar, por otro lado, que si se distingue entre caudales de baja intensidad (inferiores a $300 \mathrm{l} \mathrm{s}^{-1}$ ) y crecidas de media y alta intensidad (superiores a $300 \mathrm{I} \mathrm{s}^{-1}$ ), estas últimas se hallan muy positivamente relacionadas con la precipitación $\left(\mathrm{r}^{2}=0.44 ; \mathrm{p}=0.018\right)$, mientras que las primeras son totalmente indepen-

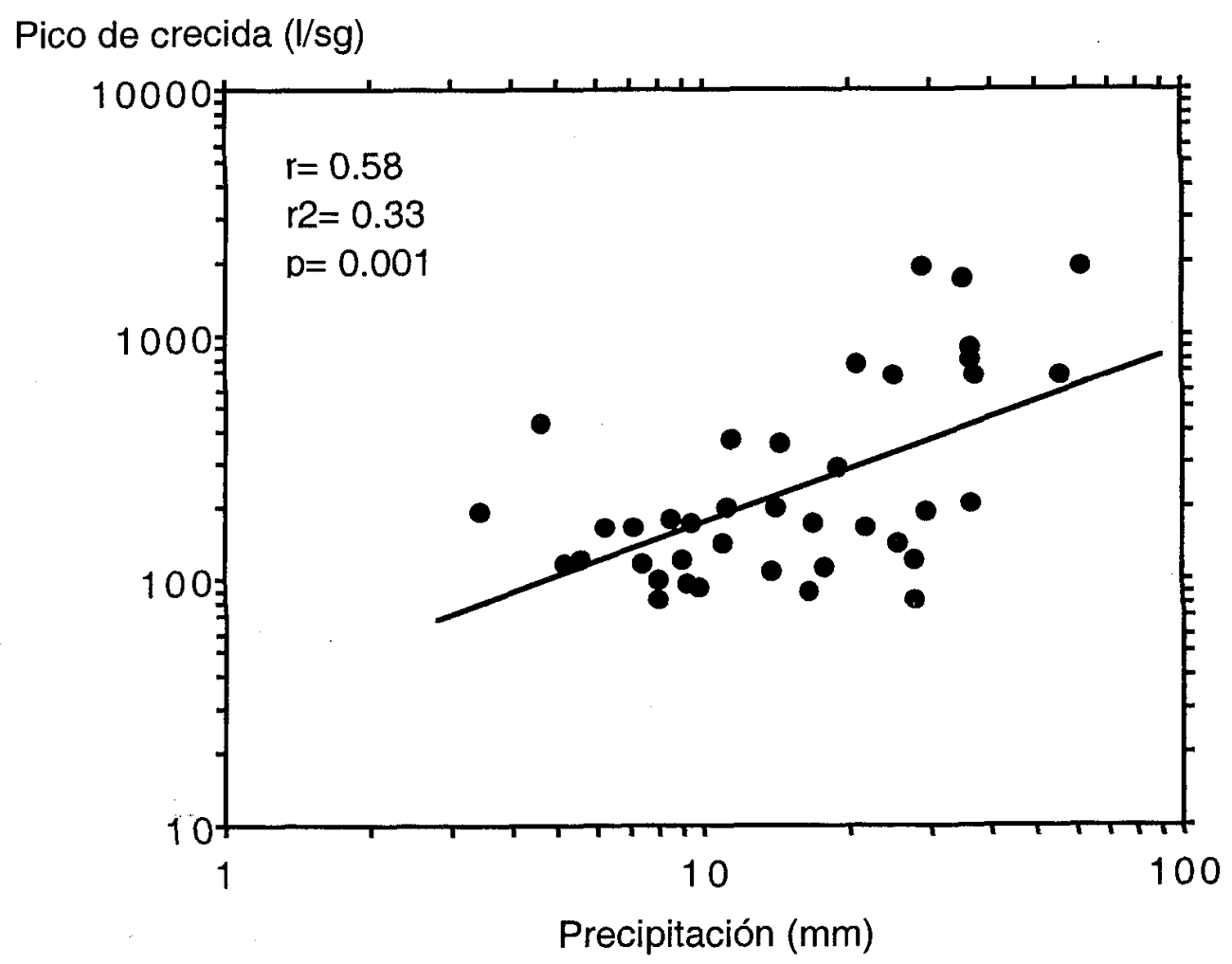

Fig. 4. Relaciones entre la precipitación y el pico de caudal de cada crecida en la cuenca de Arnás (Relationships between precipitation and peak flows of each flood in the Arnás catchment). 
dientes de la precipitación $\left(\mathrm{r}^{2}=0.020 ; \mathrm{p}=0.537\right)$. Este resultado tiene mucho valor porque, en la práctica, las crecidas más bajas contribuyen a reducir la relación existente entre precipitación y crecida.

Dado que las relaciones entre precipitación y pico de crecida ofrecen, en general, resultados muy pobres, se decidió tener en cuenta los residuales de tales relaciones, considerándolos como variable dependiente, y se han correlacionado con la precipitación registrada inmediatamente antes de cada avenida $(3,7,15 \mathrm{y}$ 21 días). Se trata, en definitiva, de comprobar si el estado antecedente de la cuenca contribuye a explicar la dispersión de puntos respecto a la recta de regresión de la Fig. 4. Los resultados son, de nuevo, decepcionantes. Si se tienen en cuenta todos los eventos, las regresiones muestran bajos coeficientes de determinación. La Tabla 4 muestra que el valor de $\mathrm{r}^{2}$ es significativo en el caso de la precipitación registrada 3 y 7 días antes de la crecida para un nivel de significación del $95 \%$. De hecho, la Tabla 4 viene a demostrar que la precipitación registrada antes de cada crecida tiene cada vez menos peso en la explicación de esa crecida a medida que aumenta la escala temporal considerada. Es decir, la lluvia caída en los 3 días anteriores tiene mayor influencia que la caída en los 7 dias anteriores y mucha mayor que la caída 15 y 21 días antes.

Si de nuevo se consideran por separado las crecidas de estación fría y cálida, resulta interesante comprobar que en el caso de la estación fría los resultados son relativamente buenos, con $\mathrm{r}^{2}=0.42(\mathrm{p}=.002)$ para la correlación entre los residuales y la precipitación registrada 3 días antes (Fig. 5), y $\mathrm{r}^{2}=0.48(\mathrm{p}=0.002)$ en el caso

Tabla 4. Coeficientes de determinación y niveles de significación entre los residuales de la relación precipitación/pico de crecida y la precipitación caída 3,7,15 y 21 días antes de cada crecida.

Variable dependiente: Residuales

\begin{tabular}{|c|c|c|c|c|c|c|}
\hline & \multicolumn{2}{|c|}{ Todos los eventos } & \multicolumn{2}{c|}{ Estación fría } & \multicolumn{2}{c|}{ Estación cálida } \\
\hline & $\mathrm{r}^{2}$ & $\mathrm{p}$ & $\mathrm{r}^{2}$ & $\mathrm{p}$ & $\mathrm{r}^{2}$ & $\mathrm{p}$ \\
\hline V1 & 0.31 & 0.001 & 0.42 & 0.002 & 0.16 & 0.229 \\
\hline V2 & 0.23 & 0.006 & 0.48 & 0.002 & 0.02 & 0.667 \\
\hline V3 & 0.13 & 0.065 & 0.26 & 0.054 & 0.11 & 0.297 \\
\hline V4 & 0.11 & 0.085 & 0.26 & 0.050 & 0.02 & 0.701 \\
\hline
\end{tabular}

V1: Precipitación 3 días antes de la crecida (mm) V2: Precipitación 7 días antes de la crecida $(\mathrm{mm})$ V3: Precipitación 15 días antes de la crecida $(\mathrm{mm})$ V4: Precipitación 21 días antes de la crecida $(\mathrm{mm})$ 
de las lluvias registradas 7 días antes. Es importante también tener en cuenta que las lluvias caídas 15 y 21 días antes son prácticamente significativas a nivel del 90 \%. En cambio, en el caso de la estación cálida no se ha encontrado ninguna relación significativa que contribuya a explicar los residuales.

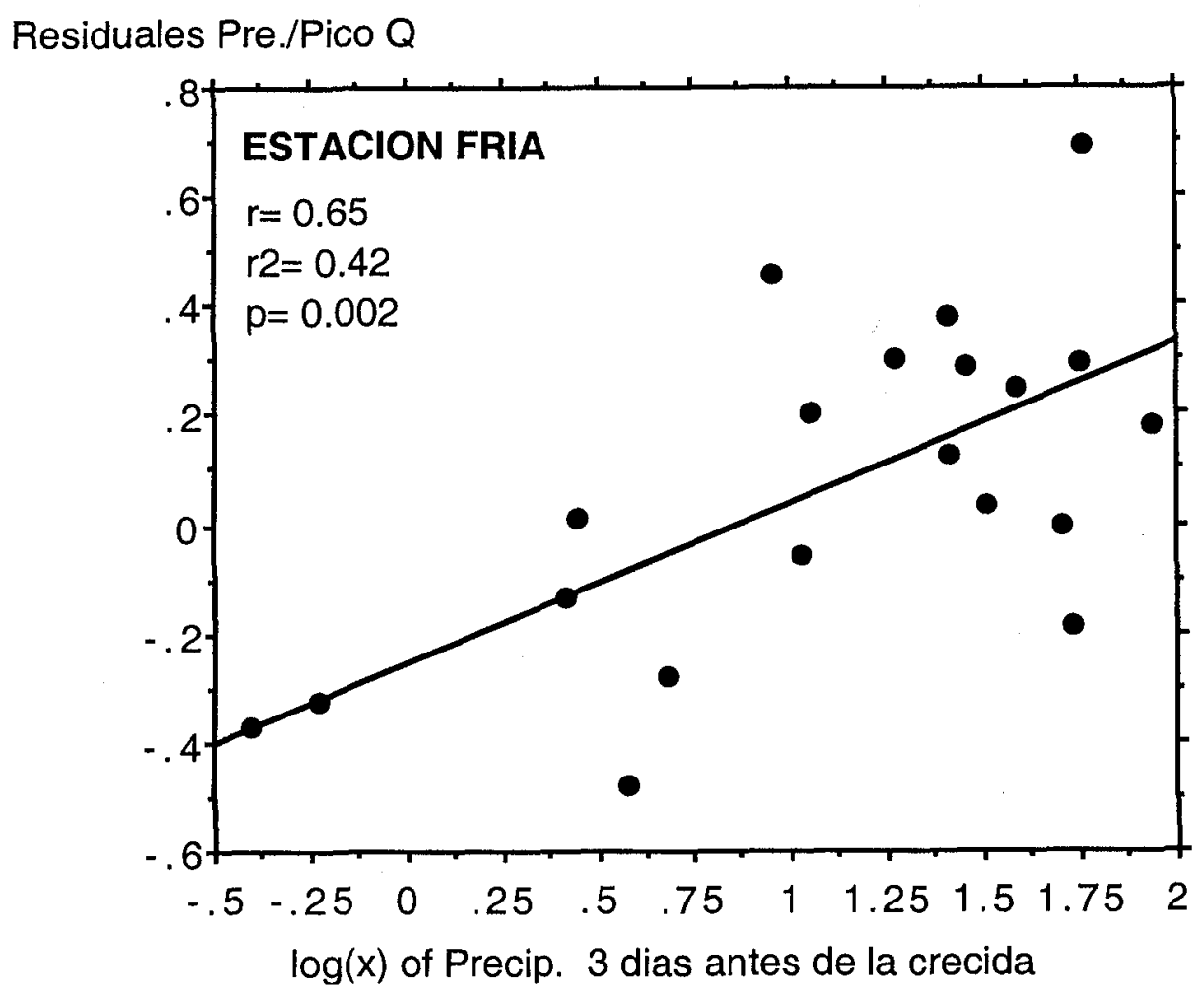

Fig. 5. Relaciones entre los residuales de la regresión precipitación/picos de crecida y la precipitación registrada durante los 3 dias anteriores a cada crecida en estación fría (Relationships between the residuals from the regression precipitation/peak flow and the precipitation recorded 3 days before each flood in cold season).

Resultados muy similares se han obtenido para las relaciones entre el volumen de caudal correspondiente a cada crecida y distintas variables pluviométricas (Tabla 5). La precipitación total sigue siendo el factor más importante (en este caso con más argumentos), y la estación fría muestra, una vez más, mejores relaciones que si se consideran todas las crecidas. Las correspondientes a la estación cálida tienen de nuevo un comportamiento aparentemente aleatorio.

Finalmente, la Tabla 6 muestra los resultados obtenidos con la regresión múltiple paso a paso, utilizando el pico de crecida y el volumen total de agua exportado en cada crecida como variables dependientes. En ambos casos dos variables 
Tabla 5. Coeficientes de determinación y niveles de significación entre el volumen de escorrentía y distintas variables pluviométricas

Variable dependiente: Volumen total de agua (1)

\begin{tabular}{|l|c|c|c|c|c|c|}
\hline & \multicolumn{2}{|c|}{ Todos los eventos } & \multicolumn{2}{c|}{ Estación fría } & \multicolumn{2}{c|}{ Estación cálida } \\
\hline & $\mathrm{r}^{2}$ & $\mathrm{p}$ & $\mathrm{r}^{2}$ & $\mathrm{p}$ & $\mathrm{r}^{2}$ & $\mathrm{p}$ \\
\hline V1 & 0.34 & $<0.001$ & 0.48 & $<0.001$ & 0.14 & 0.173 \\
\hline V2 & 0.00 & 0.705 & 0.23 & 0.017 & 0.00 & 0.880 \\
\hline V3 & 0.01 & 0.598 & 0.24 & 0.014 & 0.01 & 0.797 \\
\hline V4 & 0.04 & 0.292 & 0.17 & 0.066 & 0.18 & 0.197 \\
\hline V5 & 0.10 & 0.081 & 0.29 & 0.026 & 0.04 & 0.516 \\
\hline V6 & 0.00 & 0.811 & 0.09 & 0.280 & 0.03 & 0.582 \\
\hline V7 & 0.01 & 0.716 & 0.09 & 0.278 & 0.18 & 0.167 \\
\hline
\end{tabular}

Todas las variables han sido reconvertidas a sus correspondientes valores logarítmicos.

V1: Precipitación total $(\mathrm{mm})$

V2: Precipitación máxima en 30 minutos

V3: Precipitación máxima en 60 minutos

V4: Precipitación 3 días antes de la crecida $(\mathrm{mm})$

V5: Precipitación 7 días antes de la crecida $(\mathrm{mm})$

V6: Precipitación 15 días antes de la crecida (mm)

V7: Precipitación 21 días antes de la crecida (mm)

Tabla 6. Análisis de regresión múltiple paso a paso (todos los valores, logarítmicos)

Variable dependiente: Peak de caudal ( $1 / \mathrm{sg})$

Variables independientes: $\quad$ Precipitación total $(\mathrm{mm})$

Máxima precipitación en 30 minutos $(\mathrm{mm})$

Máxima precipitación en 60 minutos $(\mathrm{mm})$

Precipitación 3 días antes del evento $(\mathrm{mm})$

Precipitación 7 días antes del evento $(\mathrm{mm})$

Precipitación 15 días antes del evento $(\mathrm{mm})$

Precipitación 21 días antes del evento $(\mathrm{mm})$

Orden de entrada de las variables:

Paso 1 Precipitación total

Coeficiente de determinación $28 \%$

Paso 2 Precipitación 3 días antes del evento $(\mathrm{mm})$ Coeficiente de determinación 54\%

Variable dependiente: Volumen total de escorrentía (litros)

Variables independientes: las mismas que en el caso anterior

Orden de entrada de las variables:

Paso 1 Precipitación total

Coeficiente de determinación 28\%

Paso 2 Precipitación 7 días antes del evento (mm) Coeficiente de determinación $45 \%$ 
independientes explican más del 70\% de la varianza, mientras el resto de las variables contribuyen con bajos coeficientes de determinación. En el caso de los picos de crecida las variables más importantes son la precipitación total y la cantidad de lluvia registrada 3 días antes de cada crecida. En el caso del volumen total de cada crecida, las variables más determinantes han sido la precipitación total y la cantidad de precipitación registrada en los 7 días anteriores.

\section{Discusión y conclusiones}

La cuenca de Arnás responde rápidamente a casi cualquier intensidad de precipitación, debido a su pequeño tamaño, fuertes pendientes y ausencia de una densa cubierta forestal (ver García Ruiz et al., 1980). La ladera solana, con pendientes más fuertes, posee suelos de escasa potencia y con tasas de infiltración de medias a bajas, lo que favorece una rápida respuesta hidrológica. La umbría, de pendientes más suaves, cuenta con un suelo más profundo y buena permeabilidad (García Ruiz et al., 1999), si bien su mayor humedad puede acelerar los procesos de saturación (Fig. 3), facilitando así el que gran parte de la cuenca contribuya al hidrograma de crecida durante tormentas prolongadas.

Sin embargo, la cantidad total de precipitación durante cada evento individual apenas explica la intensidad de los picos de crecida, que muestran una variabilidad muy alta. Resultados similares se han obtenido en otras cuencas bajo condiciones ambientales muy diferentes (Ceballos y Schnabel, 1998; Llorens y Gallart, 1992). Es evidente que la ocurrencia de crecidas se halla estrechamente relacionada con eventos pluviométricos, dado que además la nieve caída en días aislados de invierno no llega a crear reserva suficiente como para producir verdaderas crecidas durante su fusión; pero lo que es cierto es que la respuesta hidrológica que produce cada evento pluviométrico presenta una enorme variabilidad. Los resultados obtenidos en este trabajo demuestran que existen numerosos factores que controlan la intensidad de cada crecida, además de la precipitación total por evento, y cada factor desempeña papeles diferentes a lo largo del año.

Está claro que el estado previo de humedad del suelo ejerce una notable influencia sobre el patrón de respuesta hidrológica en cada evento pluviométrico. Llorens y Gallart (1992) concluyen que en la cuenca de Vallcebre, Pirineo oriental (terrazas abandonadas), la respuesta hidrológica muestra grandes diferencias en función de las condiciones precedentes de humedad. En la cuenca de Guadalperalón, Cáceres (encinar abierto, dehesa), el caudal durante una lluvia depende mucho de la humedad antecedente del fondo del valle, de manera que si éste se halla saturado las correlaciones entre caudal y precipitación mejoran sustancialmente (Ceballos y Schnabel, 1998). Esto explica el que en la cuenca de Arnás las precipitaciones registradas durante los 3 y los 7 días anteriores a cada crecida contribuyen a explicar -aunque moderadamente- la intensidad de las crecidas. Por 
el contrario, si se analiza la influencia de las precipitaciones registradas en un plazo temporal más amplio, entonces no se ha podido detectar ninguna influencia.

Este trabajo ha demostrado que las relaciones entre precipitación y caudal de crecida se ajustan mejor durante la estación fría, debido precisamente al efecto de las precipitaciones anteriores (Tablas 3 y 4 y Fig. 5) y de la evapotranspiración. Durante la estación cálida el impacto de las precipitaciones registradas antes de cada crecida no es relevante debido a la intensa evapotranspiración y a la mayor separación temporal existente entre cada evento, lo que reduce su influencia sobre eventos posteriores. Por ello, no se ha podido obtener una correlación significativa entre precipitación y caudal. De hecho es bien conocido que las primeras lluvias tras el verano apenas generan escorrentía y lo mismo sucede con algunas tormentas de verano, que en otras cuencas pirenaicas pueden no generar escorrentía a la salida de las mismas incluso con registros superiores a $50 \mathrm{~mm}$ en 1 hora (Llorens et al., 1997). El diferente comportamiento de las crecidas de estación cálida y fría lleva a Gallart et al. (1998) a afirmar que los caudales de crecida en tiempo húmedo pueden ser fácilmente simulados, porque se ajustan a relaciones lógicas y poco complejas, mientras que las pequeñas crecidas de verano y la primera crecida de otoño después de un periodo seco son muy difíciles de modelizar.

Las crecidas inferiores a $3001 \mathrm{~s}^{-1}$ no muestran ninguna relación con la precipitación; es decir, han podido ser generadas por una precipitación de muy variable volumen, dependiendo del estado de humedad del suelo. En cambio, las crecidas más relevantes se hallan asociadas a las mayores precipitaciones. Es evidente que este comportamiento tan irregular es un notable factor de incertidumbre cuando se relacionan todas las crecidas con sus correspondientes precipitaciones.

El análisis de regresión múltiple paso a paso no contribuye a definir mejor el problema. Tanto en el caso del pico de crecida como en el del volumen total de escorrentía, dos variables absorben un elevado coeficiente de determinación, siendo en ambos casos la precipitación total el factor más importante. La segunda variable confirma que la precipitación caída en los 3 o en los 7 días anteriores a cada crecida desempeña un papel importante en la respuesta hidrológica de la cuenca. En todo caso, puede parecer sorprendente que la intensidad de la precipitación juegue un papel relativamente limitado, pero este resultado coincide con el obtenido por otros autores (Gallart et al., 1994).

\section{Agradecimientos}

Este trabajo se ha realizado con el apoyo de los siguientes proyectos de investigación: "Water resource management in a changing environment: The impact of sediment on sustainability" (WARMICE, ENV4-CT98-0789), "Validating hydrological models using process studies and internal data from research basins" (VAHMPIREn ENV4-CT95-0134) y "Debrisfall assessment in mountain catchments 
FACTORES EN LA GENERACIÓN DE CRECIDAS EN UNA CUENCA DE CAMPOS ABANDONADOS

for local end-users" (DAMOCLES, EVG1-1999-00027P), financiados por la Comisión Europea, y "Producción de escorrentía y sedimento como consecuencia de los cambios de uso del suelo en áreas de montaña" (AMB96-0401) y "Estaciones permanentes para el estudio de procesos hidrológicos en ambientes mediterráneos" (HID98-1056-Co2-01), financiados por la CICYT.

\section{Referencias bibliográficas}

Arnáez, J., García Rutz, J.M., Martí Bono, C., Bordonaba, A., Errea, M.P. y White, S., 1998: Avenidas y transporte de sedimentos en una pequeña cuenca de montaña media del Pirineo Central. In Investigaciones recientes de la Geomorfología española (A. Gómez Ortiz y F. Salvador, eds.), Sociedad Española de Geomorfología, pp.161-170, Granada.

Batalla, R.J., SAlA, M. y Werrity, A., 1995: Sediment budget focused in solid material transport in a subhumid Mediterranean drainge basin. Z. Geomorph., 39 (2): 249-264.

CAmarasa, A.M., 1990: Génesis de avenidas en pequeñas cuencas semiáridas: La rambla de Poyo (Valencia). Cuadernos de Geografía, 48: 81-104.

Ceballos, A. y Schnabel, S., 1998: Hydrological behaviour of a small catchment in the debesa landuse system (Extremadura, SW Spain). Journal of Hydrology, 210: $146-160$.

Dunne, T. y LeOpold, L.B., 1978: Water in environmental planning. Freeman \& Co., 818 pp., San Francisco.

Gallart, F. y Llorens, P., 1996: Los efectos hidrológicos de la recuperación del bosque en áreas de montaña. In Erosión y recuperación de tierras en áreas marginales (T. Lasanta y J.M. García Ruiz, eds.), Instituto de Estudios Riojanos y Sociedad Española de Geomorfología, pp. 73-78, Logroño.

GaLlarT, F., LLORENS, P. y LATRON, J., 1994: Studying the role of old agricultural terraces on runoff generation in a small Mediterranean mountainous basin. Journal of Hydrology, 159: 291-303.

Gallart, F., Llorens, P., Latron, J., Regüés, D. y Salvany, C., 1998: Las cuencas experimentales de Vallcebre (I): funcionamiento hidrológico. In Investigaciones recientes de la Geomorfología española (A. Gómez Ortiz y F. Salvador, eds.), Sociedad Española de Geomorfología, pp. 187-192, Granada.

GARCÍA RUIZ, J.M. y GALLART, F., 1997: Las cuencas experimentales como base para el estudio de la erosión y la desertificación. In El paisaje mediterráneo a través del espacio y del tiempo. Implicaciones en la desertificación (J.J. Ibáñez, B.L. Valero y C. Machado, eds.), Geoforma Ediciones, pp. 221-238, Logroño.

García Ruiz, J.M., Puigdefábregas, J. y Creus, J., 1980: Influencia de las características físicas de las cuencas hidrográficas en la frecuencia e intensidad de crecidas. Cuadernos de Investigación Geográfica, 6: 19-36. 
J. ARNÁEZ - C. MARTÍ BONO - S. BEGUERÍA - A. LORENTE - M.P. ERREA - J.M. GARCÍA RUIZ

García Ruiz, J.M., Puigdefábregas, J. y Creus, J., 1985: Los recursos hidricos superficiales del Alto Aragón. Instituto de Estudios Altoaragoneses, 224 pp., Huesca. García Ruiz, J.M., White, S., NAVAS, A. y Errea, M.P., 1999: CSIC/IPE contribution to the Vahmpire Project. In Validating bydrological models using process studies and internal data from research basins: Tools for assessing bydrological impacts of environmental change (F. Gallart, S. White y P. Llorens, eds.), European Commission, pp. 91-134, Barcelona.

González, C., García Ruiz, J.M., Martí, C., White, S., Errea, M.P. y Arnáez, J., 1997: Sediment sources in a small, abandoned farmland catchment, Central Spanish Pyrenees. Physics and Chemistry of the Earth, 22 (3-4): 291-293.

LLORENS, P. Y GALLART, F., 1992: Small basin response in a Mediterranean mountainous abandoned farming area: Research design and preliminary results. Catena, 19 (3-4): 309-320.

LLORENS, P., 1993: Hydrological implications of afforestation of abandoned lands: water balance simulation of a small Mediterranean basin. Acta Geológica Hispánica, 28: 131-138.

Llorens, P., Queralt, I., Plana, F. y Gallart, F., 1997: Studying solute and particulate sediment transfer in a small Mediterranean mountainous catchment subject to land abandonment. Earth Surface Processes and Landforms, 22: 1027-1035.

Lorente, A., Martí Bono, C., Beguería, S., Arnáez, J. y García Ruiz, J.M., en prensa: La exportación de sedimento en suspensión en una cuenca de campos abandonados, Pirineo Central. Cuaternario y Geomorfología.

Martínez Castroviejo, R., García Ruiz, J.M., Díez, J.C. y Alvera, B., 1991: Coarse sediment transport in an experimental high mountain catchment of Central Pyrenees. Z. Geomorph. Suppl. Bd., 83: 105-114.

Regǘtés, D., Gallart, F., Llorens, P., Latron, J. y Soler, M., 1998: Las cuencas experimentales de Vallcebre (II): dinámica de sedimentos. In Investigaciones recientes de la Geomorfología española (A. Gómez Ortiz y F. Salvador, eds.), Sociedad Española de Geomorfología, pp.259-264, Granada.

White, S., García Ruiz, J.M., Martí, C., Alvera, B. y Del Barrio, G., 1997: Sediment transport in a high mountain catchment in the Central Spanish Pyrenees. Physics and Chemistry of the Earth, 22 (3-4): 377-380. 